\title{
Soft Decoding Techniques for Codes and Lattices, Including the Golay Code and the Leech Lattice
}

\author{
JOHN H. CONWAY AND N. J. A. SLOANE, FELLOW, IEEE
}

\begin{abstract}
Two kinds of algorithms are considered. 1) If $\mathscr{C}$ is a binary code of length $n$, a "soft decision" decoding algorithm for $\mathscr{C}$ changes an arbitrary point of $R^{n}$ into a nearest codeword (nearest in Euclidean distance). 2) Similarly, a decoding algorithm for a lattice $\Lambda$ in $R^{n}$ changes an arbitrary point of $R^{n}$ into a closest lattice point. Some general methods are given for constructing such algorithms, and are used to obtain new and faster decoding algorithms for the Gosset lattice $E_{8}$, the Golay code and the Leech lattice.
\end{abstract}

\section{INTRODUCTION}

$\mathrm{L}$ ET $\mathscr{C}$ be an $[n, k]$ binary code. We regard the codewords as points of $n$-dimensional Euclidean space $\boldsymbol{R}^{n}$, and wish to find a "soft decision" decoder for $\mathscr{C}$ (also called an "analog" or "maximum likelihood" decoder). By this we mean an algorithm which, when presented with an arbitrary point $x$ of $\boldsymbol{R}^{n}$, will find a codeword $u \in \mathscr{C}$ that minimizes dist $(x, u)$, the distance being Euclidean distance. Soft decision decoding has been investigated by many distinguished information theorists over the yearssee [1]-[12], [14], [31]-[35], [40]-[45], [51], [52], [54], [58], [60], [62], [66]-[69]. However, the majority of these papers study decoding algorithms that only perform correctly most of the time. For example, Hackett's decoding algorithm [42] for the Golay code is "only a few tenths of a decibel different from ideal correlation detection." In the present paper we are only interested in algorithms that always find a closest codeword or lattice point.

The decoding problem for a lattice $\Lambda$ in $\boldsymbol{R}^{n}$ is similar. We wish to find an algorithm which, when presented with an arbitrary point of $\boldsymbol{R}^{n}$, will find a lattice point $u \in \Lambda$ that minimizes dist $(x, u)$. Decoding algorithms for several classes of lattices were given in [22], [28].

In Section II we collect together all the methods we know for constructing decoding algorithms (of the type just mentioned, that always give the correct answer) for codes and lattices. Most of these methods were known already, although some are new. We then apply these methods to obtain improved decoding algorithms for the $E_{8}$ lattice

Manuscript received October 10, 1984; revised June 21, 1985.

J. H. Conway is with the Department of Pure Mathematics and Mathematical Statistics, University of Cambridge, Cambridge CB2 1SB, England.

N. J. A. Sloane is with the Mathematical Sciences Research Center, Room 2C-376, Bcll Laboratories, Murray Hill, NJ 07974.

IEEE Log Number 8406089.
(Section II, paragraph 13)), the Golay code (Section III), and the Leech lattice (Section IV).

Potential applications of these algorithms are to channel coding and vector quantizing (see the references already mentioned, and [12], [13], [21], [27], [36], [37], [64]). It is worth mentioning that there is already a considerable literature devoted to "hard decision" or conventional binary decoding of the Golay code ([50, ch. 16, § 9], [5], [31], [39], [70], [71]).

Notation: Two codes or lattices $\mathscr{A}$ and $\mathscr{B}$ are geometrically similar if one can be obtained from the other by (possibly) a translation, rotation, reflection, and change of scale. The direct sum $[50$, p. 76] of two codes or lattices $\mathscr{A}$ and $\mathscr{B}$ is written $\mathscr{A} \oplus \mathscr{B}$. The componentwise product of two vectors $u$ and $v$ is written $u * v$.

\section{Fast Decoding Algorithms}

We first consider codes. Let $\mathscr{C}$ be an $[n, k]$ binary code. It is often more convenient to write the codewords as vectors of +1 's and -1 's rather than 0 's and 1's. Note that if

$$
w=u+v \quad \text { in } 0,1 \text { notation }
$$

then

$$
w=u * v \quad \text { in }+1,-1 \text { notation. }
$$

The $+1,-1$ notation allows us to replace distance calculations with inner product calculations. For, if $x \in \boldsymbol{R}^{n}$ and $u \in \mathscr{C}$

$$
\begin{aligned}
\operatorname{dist}^{2}(x, u) & =(x-u) \cdot(x-u) \\
& =x \cdot x-2 x \cdot u+u \cdot u \\
& =x \cdot x-2 x \cdot u+n .
\end{aligned}
$$

Thus finding a closest codeword to $x$ is equivalent to finding a codeword (in $+1,-1$ notation) that has the largest inner product with $x$.

The following codes, and those geometrically similar to them, are most of the codes we know that have a fast "soft decision" decoding algorithm as described in Section I. We give rough estimates ${ }^{1}$ of the number of arithmetic steps

\footnotetext{
${ }^{1}$ As the automobile advertisements say, use these figures for comparison only. The actual running time will depend on the relative speeds of addition and multiplication, etc., and will probably be greater than the figures given here. We have tried, however, to evaluate all the algorithms in a uniform manner.
} 
(additions, multiplications, etc.) required. "Fast" means that the algorithm is substantially faster than the following direct search method.

1) Any small code may be decoded by a direct search. For an $[n, k]$ code we compute the inner product of the given vector $x$ with every codeword and choose the closest. Assuming we have precomputed a list of the codewords, this requires roughly $2 n \cdot 2^{k}$ steps and is therefore only applicable to small codes. If the code contains the vector $(-1,-1, \cdots,-1)$-i.e., the all 1 's vector in 0,1 notation - the codewords come in pairs $+u$ and $-u$, and the number of steps drops to $n 2^{k}$.

2) A first-order Reed-Muller code, with parameters $[n=$ $\left.2^{m}, k=m+1\right]$, may be efficiently decoded using the fast Hadamard transform (the so-called Green machine described in [40], [41], [58], [50, ch. 14]). This computes the $2^{m+1}$ inner products $x \cdot u, u \in \mathscr{C}$, in about $m 2^{m}$ steps. It is worth pointing out that a first-order Reed-Muller code is geometrically similar to an octahedron ( $\beta_{n}$ in Coxeter's notation [30]). For example, the codewords of the code of length 4 shown in Fig. 1(a) when multiplied by the orthogonal matrix of Fig. 1(b) become the eight vertices

$$
( \pm 2,0,0,0), \cdots,(0,0,0 \pm 2)
$$

of a four-dimensional octahedron.

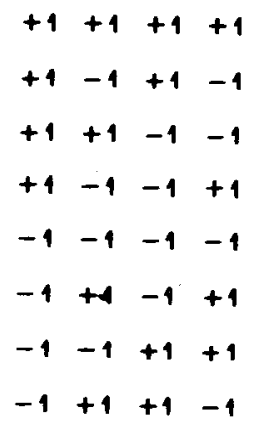

(a)

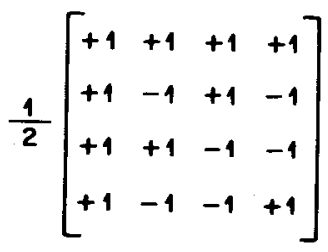

(b)
Fig. 1. (a) First-order Reed-Muller code of length 4. (b) Orthogonal matrix.

A $\left[2^{m}-1, m\right]$ simplex code $[50$, p. 30$]$ may be decoded by a straightforward modification of the fast Hadamard transform. (Set one of the inputs, say the last, to zero, and only calculate the linear combinations in which the last variable occurs with a + sign.) This code is geometrically similar to a simplex ( $\alpha_{n}$ in the notation of [30]).

3) The universe code $\mathscr{F}_{n}$, consisting of all binary vectors of length $n$, may be decoded in just $n$ steps. To decode $x=\left(x_{1}, \cdots, x_{n}\right)$, we simply replace each $x_{i}$ by $\operatorname{sgn}\left(x_{i}\right)$, where

$$
\begin{aligned}
\operatorname{sgn}(x) & =+1, & & \text { if } x \geq 0, \\
& =-1, & & \text { if } x<0 .
\end{aligned}
$$

This is more efficient even than the fast Hadamard transform of 2), since it selects one out of $2^{n}$ codewords in $n$ steps, whereas the fast Hadamard transform selects one of $2 n$ codewords in $n \log _{2} n$ steps. $\mathscr{F}_{n}$ is geometrically similar to a cube $\left(\gamma_{n}\right.$ in the notation of [30]), the reciprocal polytope to the octahedron.
Of course not many interesting codes contain $\mathscr{F}_{n}$ itself as a subcode. But codes geometrically similar to $\mathscr{F}_{n}$ are quite common. For example, the Golay code $\mathscr{G}_{24}$ (see Section III) contains a subcode of dimension 3 generated by

$$
\left[\begin{array}{lll}
11111111 & 00000000 & 00000000 \\
00000000 & 11111111 & 00000000 \\
00000000 & 00000000 & 11111111
\end{array}\right]
$$

in 0,1 notation, i.e., by

$$
-1^{8}+1^{16},+1^{8}-1^{8}+1^{8},+1^{16}-1^{8} \quad \text { in } \pm 1 \text { notation. }
$$

This subcode is geometrically similar to $\mathscr{F}_{3}$ and may be decoded as follows. Given $x=\left(x_{1}, \cdots, x_{24}\right)$, we decode it as

$$
u=a a \cdots a b b \cdots b c c \cdots c,
$$

where $a, b$, and $c$ each appear eight times, and

$$
\begin{aligned}
& a=\operatorname{sgn}\left(x_{1}+\cdots+x_{8}\right), \\
& b=\operatorname{sgn}\left(x_{9}+\cdots+x_{16}\right), \\
& c=\operatorname{sgn}\left(x_{17}+\cdots+x_{24}\right) .
\end{aligned}
$$

Furthermore, we see from (5) that the maximal inner product $x \cdot u$ is given by

$$
\left|\sum_{i=1}^{8} x_{i}\right|+\left|\sum_{i=9}^{16} x_{i}\right|+\left|\sum_{i=17}^{24} x_{i}\right| .
$$

A slightly more general family of codes can be decoded in the same way. Let $T_{a}$ denote the $[a, 1]$ repetition code of length $a$. Then

$$
T_{a_{1}} \oplus T_{a_{2}} \oplus \cdots \oplus T_{a_{n}}
$$

although in general not geometrically similar to $\mathscr{F}_{n}$, can be decoded by an obvious modification of the preceding algorithm.

4) The even weight code $\mathscr{E}_{n}$, with parameters $[n, k=$ $n-1$, consists of all 0,1 vectors with an even number of ones and may be decoded in about $2 n$ steps. To decode $x=\left(x_{1}, \cdots, x_{n}\right)$, we first replace each $x_{i}$ by $\operatorname{sgn}\left(x_{i}\right)$. If there are an even number of minus signs we stop, but if there are an odd number we reverse the sign on an $x_{i}$ of smallest magnitude. $\mathscr{E}_{n}$ is geometrically similar to a hemicube $\left(h_{\gamma n}\right.$ in the notation of $[30$, p. 155]).

Example 1: The Golay code contains a [24,5] subcode with generator matrix

$\left[\begin{array}{llllll}1111 & 1111 & 0000 & 0000 & 0000 & 0000 \\ 1111 & 0000 & 1111 & 0000 & 0000 & 0000 \\ 1111 & 0000 & 0000 & 1111 & 0000 & 0000 \\ 1111 & 0000 & 0000 & 0000 & 1111 & 0000 \\ 1111 & 0000 & 0000 & 0000 & 0000 & 1111\end{array}\right]$,

the sextet code (cf. [15]), which is geometrically similar to $\mathscr{E}_{6}$. Since this code plays a key role in Section III, we give the precise decoding algorithm. To decode $x=\left(x_{1}, \cdots\right.$, $x_{24}$ ) we first compute

$$
s_{\mathrm{I}}:=\sum_{i=1}^{4} x_{i}, s_{\mathrm{II}}:=\sum_{i=5}^{8} x_{i}, \cdots, s_{\mathrm{VI}}:=\sum_{i=21}^{24} x_{i},
$$


and

$$
u_{\mathrm{I}}:=\operatorname{sgn}\left(s_{\mathrm{I}}\right), \cdots, u_{\mathrm{VI}}:=\operatorname{sgn}\left(s_{\mathrm{VI}}\right)
$$

If an odd number of the $u_{N}$ are negative we change the sign of a $u_{N}$ for which $\left|s_{N}\right|$ is minimal. Then $x$ is decoded as

$$
u:=u_{\mathrm{I}} u_{\mathrm{I}} u_{\mathrm{I}} u_{\mathrm{I}} u_{\mathrm{II}} u_{\mathrm{II}} u_{\mathrm{II}} u_{\mathrm{II}} \cdots u_{\mathrm{VI}} u_{\mathrm{VI}} u_{\mathrm{VI}} u_{\mathrm{VI}},
$$

and the maximal inner product is given by

$$
x \cdot u=\left|s_{\mathrm{I}}\right|+\cdots+\left|s_{\mathrm{vI}}\right|
$$

if an even number of the $u_{N}$ in (9) are negative, otherwise by

$$
x \cdot u=\left|s_{\mathrm{I}}\right|+\cdots+\left|s_{\mathrm{VI}}\right|-2 \min _{N}\left|s_{\mathrm{N}}\right| \cdot
$$

Example 2: The $[2 n, n-1]$ code $d_{n}$ generated by $111100 \cdots, 0011110 \cdots, 000011110 \cdots, \cdots[57$, p. 320] is geometrically similar to $\mathscr{E}_{n}$.

Remark: Permutation codes [6], [7], [62] are a class of (in general) nonbinary codes that include first-order Reed-Muller codes, simplex codes, $\mathscr{F}_{n}$ and $\mathscr{E}_{n}$ as special cases, and may also be decoded rapidly.

5) Codes with small redundancy may be regarded as trellis codes, as pointed out by Solomon [11], [66] and Wolf [69], and therefore can be efficiently decoded by the Viterbi algorithm [35], [68]. For an $[n, k]$ code, the trellis has $2^{n-k}$ states, and the number of decoding steps is roughly $4 n 2^{n k}$.

6) Direct sums of codes on this list can also be decoded easily. To decode $\mathscr{A} \oplus \mathscr{B}$, for example, we apply the decoder for $\mathscr{A}$ to the first part of $x$ and the decoder for $\mathscr{B}$ to the second part. The number of steps is the sum of the numbers of steps for decoding $\mathscr{A}$ and $\mathscr{B}$.

7) Supercodes: If $\mathscr{B}$ is one of the foregoing codes, and $\mathscr{C}$ contains $\mathscr{B}$ as a subcode of small index, then $\mathscr{C}$ can also be decoded easily. Let us write

$$
\mathscr{C}=\bigcup_{j=0}^{t-1}\left(\rho^{(j)}+\mathscr{B}\right)
$$

where $t:=|\mathscr{C}| /|\mathscr{B}|$ is the index of $\mathscr{B}$ in $\mathscr{C}$, and the $\rho^{(j)}$ $(j=0, \cdots, t-1)$ are coset representatives for $\mathscr{B}$ in $\mathscr{C} . \mathscr{C}$ is called a supercode of $\mathscr{B}$. Equation (12) applies to 0,1 vectors, while in terms of $+1,-1$ vectors we have

$$
\mathscr{C}=\bigcup_{j=0}^{t-1} \rho^{(j)} * \mathscr{B}
$$

(compare (1),(2)). Finding the largest inner product of $x$ with the vectors in $\rho^{(j)} * \mathscr{B}$ is equivalent to finding the largest inner product of $\rho^{(j)} * x$ with the vectors in $\mathscr{B}$. So provided $t$ is not too large, we may decode $x$ as follows.

$$
\text { Compute } y^{(j)}:=\rho^{(j)} * x \text {, and }
$$

use the decoder for $\mathscr{B}$ to find $u^{(j)}:=$ the closest vector in $\mathscr{B}$ to $y^{(/)}$and

$$
i p^{(j)}:=u^{(j)} \cdot y^{(j)} .
$$

After doing this for $j=0,1, \cdots, t-1$, the largest $i p^{(j)}$ is the final inner product, and the corresponding $u^{(j)}$ is used to produce the decoder output, $\rho^{(j)} * u^{(j)}$.
This is similar to [22, algorithm 3]. The number of steps required is about $t$ times the number of steps to decode $\mathscr{B}$, plus $2 n t$ to compute the $y^{(j)}$ in (14). In practice this number $2 n t$ can often be greatly reduced, as we shall see in Section III.

Example: Codes formed by "gluing" several subcodes together can be decoded in this way. Fig. 2 shows a generator matrix for a typical code of this type, consisting of a direct sum of subcodes $\mathscr{B}_{1}, \mathscr{B}_{2}, \cdots$, together with certain additional generators called glue vectors. If there are $g$ additional generators, the index of the subcode $\mathscr{B}_{1} \oplus \mathscr{B}_{2} \oplus \cdots$ in the full code is $t=2^{g}$. Furthermore, the subcode generated by these $g$ glue vectors (the glue code) consists of precisely the coset representatives $\rho^{(j)}$ appearing in (12). Numerous examples of such codes may be found in [18], [56], [57].

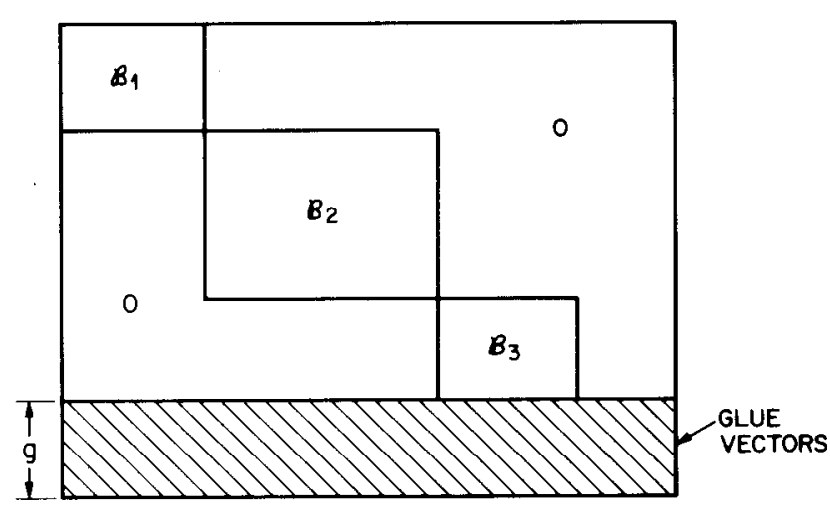

Fig. 2. Code formed by "gluing" subcodes $\mathscr{B}_{1}, \mathscr{B}_{2}, \mathscr{B}_{3}$ together.

8) Shortened codes: If an $[n, k]$ code $\mathscr{C}$ can be decoded efficiently, so can the $[n-1, k]$ code $\mathscr{B}$ obtained by deleting the last coordinate of every codeword. To decode $x=\left(x_{1}, \cdots, x_{n-1}\right)$, we simply apply the decoder for $\mathscr{C}$ to $\left(x_{1}, \cdots, x_{n-1}, 0\right)$. Since the codewords in $\mathscr{C}$ end with +1 or -1 , this will find the closest codeword to $x$.

Next we consider lattices. Given a lattice $\Lambda$ in $\boldsymbol{R}^{n}$, the decoding algorithm changes an arbitrary point of $\boldsymbol{R}^{n}$ into a closest lattice point. (We can no longer work with inner products.) The following lattices, and those geometrically similar to them, are most of the lattices we know that have a fast decoding algorithm.

9) The cubic lattice $Z^{n}$, consisting of all points $\left(u_{1}, \cdots\right.$, $u_{n}$ ) with integer coordinates, can be decoded in about $n$ steps. If $x_{i}$ is a real number, we define

$$
f\left(x_{i}\right):=\text { nearest integer to } x_{i},
$$

rounding towards zero in case of a tie, and for a vector $x=\left(x_{1}, \cdots, x_{n}\right)$ we define

$$
f(x)=\left(f\left(x_{1}\right), \cdots, f\left(x_{n}\right)\right) .
$$

Then the decoder for $\boldsymbol{Z}^{n}$ simply changes $x$ to $f(x)$ [22, sect. III].

10) The lattice $D_{n}$, consisting of all points in $Z^{n}$ whose coordinates add to an even number, can be decoded in 
about $4 n$ steps. For $x \in Z^{n}$, we define $g(x)$ to be the same as $f(x)$, except that the worst component of $x$-that furthest from an integer-is rounded the wrong way. In case of a tie, the component with the lowest subscript is rounded the wrong way. (Formal definitions of $f$ and $g$ may be found in $[22$, p. 228].)

The decoder for $D_{n}$ computes $f(x)$ and $g(x)$, and the sum $f\left(x_{1}\right)+\cdots+f\left(x_{n}\right)$ of the components of $f(x)$. If the sum is even, the output is $f(x)$, and if it is odd, the output is $g(x)$. (See [22] for a proof and an example.)

The number of steps may be estimated as follows. For each of the $n$ components of $x$, we compute a) $f\left(x_{i}\right)$ and b) the error $e_{i}=x_{i}-f\left(x_{i}\right)$, c) test if $e_{i}$ is a new worst error [needed to determine $g(x)$ ], and d) update $\Sigma f\left(x_{i}\right)$. Thus the total number of steps is about $4 n$.

11) The lattice $A_{n}$, consisting of all points in $Z^{n+1}$ whose coordinates add to zero, can be decoded in a constant times $n \log _{2} n$ steps by the algorithm given in [22, sec. VII]. As pointed out to us by A. M. Odlyzko, this can be reduced to a constant times $n$ by the following argument. If the discrepancy is $\Delta$ (see [22]), it is necessary to find the $|\Delta|$ largest (if $\Delta>0$, or smallest, if $\Delta<0$ ) of the numbers $\delta\left(x_{i}^{\prime}\right)$. This can be done in a constant times $n$ steps using the Rivest-Tarjan algorithm (see [46]). For $n=2$ and 3 there are better algorithms. $A_{2}$ is the familiar two-dimensional hexagonal lattice and is best decoded using the fact that it is the union of a rectangular lattice and a translate, as suggested by Gersho [37, p. 165], [28, p. 299]. $A_{3}$ is geometrically similar to the face-centered cubic lattice $D_{3}$ and is best decoded by the $D_{3}$ algorithm.

12) Lattices obtained from Construction $A$ : If $\mathscr{C}$ is an $[n, k]$ binary code, Construction A [48], [63] produces a lattice $\Lambda(\mathscr{C})$ in $\boldsymbol{R}^{n}$. If the codewords of $\mathscr{C}$ are written in 0,1 notation, the points of $\Lambda(\mathscr{C})$ consist of all vectors of the form

$$
c+2 z, \quad \text { for } c \in \mathscr{C}, \quad z \in Z^{n},
$$

where we regard the zeros and ones in $c$ as real numbers rather than elements of the Galois field $G F(2)$. For our present purposes we wish to write the codewords in $+1,-1$ notation, in which case the points of $\Lambda(\mathscr{C})$ consist of all vectors of the form

$$
c+4 z, \quad \text { for } c \in \mathscr{C}, z \in Z^{n} .
$$

[The set of points (18) strictly speaking no longer forms a lattice but rather is a translate of a lattice by the vector $(1,1, \cdots, 1)$.]

The following lemma makes it possible to use a decoding algorithm for $\mathscr{C}$ to decode $\Lambda(\mathscr{C})$.

Lemma: Suppose $x=\left(x_{1}, \cdots, x_{n}\right)$ lies in the cube -1 $\leq x_{i} \leq 1(i=1, \cdots, n)$. Then no point of $\Lambda(\mathscr{C})$ is closer to $x$ than the closest codeword of $\mathscr{C}$.

Proof: Suppose the contrary, and let $u=\left(u_{1}, \cdots, u_{n}\right)$ be a closest lattice point to $x$. By hypothesis some $u_{i}$ 's are neither +1 nor -1 . By subtracting a suitable vector $4 z$, we may change these coordinates to +1 or -1 (depending on their parity) to produce a point of $\Lambda(\mathscr{C})$ that is in $\mathscr{C}$, and is at least as close to $x$ as $u$ is, a contradiction.
Decoding Algorithm for $\Lambda(\mathscr{C})$ :

1) Given $x=\left(x_{1}, \cdots, x_{n}\right)$, we first reduce all $x_{i}$ to the range $-1 \leq x_{i}<3$ by subtracting a vector $4 z$.

2) Let $S$ denote the set of $i$ for which $1<x_{i}<3$. For $i \in S$, replace $x_{i}$ by $2-x_{i}$.

3) Since $x$ is now in the cube $-1 \leq x_{i} \leq 1$ ( $i=$ $1, \cdots, n)$, by the Lemma we are justified in applying the decoder for $\mathscr{C}$ to $x$, obtaining an output $c=$ $\left(c_{1}, \cdots, c_{n}\right)$, say.

4) For $i \in S$, change $c_{i}$ to $2-c_{i}$. Then $c+4 z$ is a closest point of $\Lambda(\mathscr{C})$ to the original vector $x$.

The number of steps may be estimated as follows. For each $x_{i}$ we compute

the nearest integer to $x_{i}-1$ that is a multiple

$$
\begin{aligned}
& \text { of } 4 \text {, say } 4 z_{i}, \\
& \text { the difference } d_{i}-x_{i}-4 z_{i} \text {, and } \\
& \text { if } d_{i}>2 \text {, change } d_{i} \text { to } 2-d_{i} \text { (for } i \in S \text {, say). }
\end{aligned}
$$

Then we apply the decoder for $\mathscr{C}$ to $\left(d_{i}, \cdots, d_{n}\right)$, producing $c=\left(c_{1}, \cdots, c_{n}\right)$. Finally, we

$$
\begin{aligned}
& \text { change } c_{i} \text { to } 2-c_{i} \text { for } i \in S \text {, and } \\
& \text { add } 4 z \text { to } c \text {. }
\end{aligned}
$$

The total number of steps is roughly $5 n$ plus the number to decode $\mathscr{C}$.

Many interesting sphere packings can be obtained from Construction A [29], [48], [63], the most important being the $E_{8}$ lattice-see paragraph 13 ).

Remark: Unfortunately, it does not appear possible to modify this algorithm to apply to lattices obtained from Construction B [48], [63]. (Construction B differs from Construction $\mathrm{A}$ in that in (17) and (18) $z=\left(z_{1}, \cdots, z_{n}\right)$ must satisfy $\Sigma z_{i} \equiv 0(\bmod 2)$.) If such a modification were found, it would further speed up the decoding algorithm for the Leech lattice given in Section IV.

13) The Gosset lattice $E_{8}$ : This important lattice can be constructed in several ways (see [12], [21], [22], [24]-[26], [29], [48]), one of which is to apply Construction A (see 12)) to the $[8,4]$ first-order Reed-Muller code $\mathscr{C}$. In this form $E_{8}$ consists of the vectors

$$
c+4 z, \quad c \in \mathscr{C}, \quad z \in \boldsymbol{Z}^{8},
$$

$c$ being a $+1,-1$ vector of length 8 . [As mentioned earlier, with these coordinates $E_{8}$ is not a lattice but a translate of a lattice by the vector $(1,1, \cdots, 1)$.] $\mathscr{C}$ can be decoded in about $3 \cdot 8+8=32$ steps by a fast Hadamard transform (see 2)). Therefore the algorithm given in 12) will decode this version of $E_{8}$ in roughly 72 steps. This is faster than the algorithm proposed in [22] (see paragraph 15) to follow) which requires about 104 steps.

If we require not only the closest point $u \in E_{8}$ to $x$, but also dist ${ }^{2}(x, u)$, this can be obtained at the end of step 3) of the algorithm, using (3), at the cost of about 16 additional steps to compute $x \cdot x$. If steps 1) and 2) can be carried out in advance, as will be the case when we use this algorithm in decoding the Leech lattice in Section IV, the 
number of steps to decode one $x$ drops to about 48 , or 56 if $\operatorname{dist}^{2}(x, u)$ is needed.

14) Direct sums of lattices on this list can be handled in the same way as direct sums of codes-see 6).

15) Superlattices: If $M$ is one of the foregoing lattices and $\Lambda$ contains $M$ as a sublattice of small index, then $\Lambda$ can also be decoded easily. We write

$$
\Lambda=\bigcup_{j=0}^{t-1}\left(\rho^{(j)}+M\right)
$$

where $t:=\operatorname{det} M / \operatorname{det} \Lambda$ is the index of $M$ in $\Lambda$, $\operatorname{det} M$ is the volume of a fundamental domain for $M$ (see [21], [63], [65]), and the $\rho^{(j)}(j=0,1, \cdots, t-1)$ are coset representatives for $M$ in $\Lambda$. Let $\Phi$ be a decoder for $M$.

To decode $\Lambda$ we proceed as follows. Given $x$, we calculate

$$
\begin{aligned}
y^{(j)} & :=x-\rho^{(j)} \\
z^{(j)} & :=\Phi\left(y^{(j)}\right) \\
d_{j} & :=\left(z^{(j)}-y^{(j)}\right) \cdot\left(z^{(j)}-y^{(j)}\right),
\end{aligned}
$$

for $j=0, \cdots, t-1$, and find a $j, j^{*}$ say, for which $d_{j}$ is minimized. Then the decoder output is

$$
u:=z^{\left(j^{*}\right)}+\rho^{\left(j^{*}\right)},
$$

and $d_{j^{*}}$ is the squared distance from $x$ to $u[22$, sec. V].

The number of steps required is about $t$ times the number of steps to decode $M$, plus $n(t-1)$ steps to compute the $y^{(j)}$ (in practice this can often be greatly reduced-see Section IV), plus $3 n t$ steps to compute the $d_{j}$.

Example 1: The $E_{8}$ lattice (see 13)) is also given by

$$
E_{8}=D_{8} \cup\left(\frac{1}{2} \frac{1}{2} \frac{1}{2} \frac{1}{2} \frac{1}{2} \frac{1}{2} \frac{1}{2} \frac{1}{2}\right)+D_{8},
$$

an example in which $t=2$ and $M=D_{8}$. The foregoing algorithm (proposed in [22]) therefore takes about $2 \cdot 32+$ $8+32=104$ steps. This drops to about 96 if $y^{(0)}$ and $y^{(1)}$ have been precomputed.

Example 2: The dual lattices $D_{n}^{*}$ and $A_{n}^{*}$ (the so-called Voronoi lattice of the first type, which is important for the covering problem [61]) may also be decoded by this algorithm (see [22]).

Example 3: Lattices formed by "gluing" sublattices together (in exactly the same way the codes were glued together in 7)) may be decoded by this algorithm. This includes the twenty-three 24-dimensional Niemeier lattices [53], [19], as well as many other lattices described in [19], [20].

Based on the results assembled in this section, we can now state our general strategy for finding an efficient decoding algorithm for a code or lattice: find a subcode (or sublattice) of smallest index for which a fast algorithm exists, and then use 7), 12), or 15). Algorithms for decoding the lattices $E_{6}, E_{6}^{*}, E_{7}, E_{7}^{*}, K_{12}$, and $\Lambda_{16}$ were obtained in [28] by using this strategy. For example, the Coxeter-Todd lattice $K_{12}$ contains $A_{2} \oplus \cdots \oplus A_{2}$ (six summands) as a sublattice of index 64 . In the next two sections we apply the strategy to the Golay code and the Leech lattice.

\section{Fast Decoding Algorithms for the Golay CODE}

The direct search algorithm (see Section II, paragraph 1)) for the $[24,12]$ Golay code $\mathscr{G}_{24}$ takes about $24 \cdot 2^{12}=$ 98304 steps, while the trellis decoding method of Section II, paragraph 5) is even slower. Hwang's algorithm [44], [45] for the shortened Golay code of length 23 takes roughly $24 \cdot 1376=33024$ steps. In this section we give two algorithms that are much faster. The first is based on the subcode (7), takes about 1584 steps, and is described in detail. The second is based on the subcode (4), takes about 1728 steps, and is only sketched. The second algorithm is included because it could easily be faster than the first if a different method were used to count steps.

\section{A. The First Algorithm}

There are three parts to the algorithm: the design stage, which is only performed once, and the precomputation and main stages, which are both performed each time a vector is decoded.

The Design Stage: $\mathscr{G}_{24}$ contains the $[24,5]$ subcode $\mathscr{B}$ shown in (7). As coset representatives for $\mathscr{B}$ in $\mathscr{G}_{24}$ we take the 128 words of the code defined by the following generator matrix:

$\left[\begin{array}{llllll}1100 & 1100 & 1100 & 1100 & 0000 & 0000 \\ 1010 & 1010 & 1010 & 1010 & 0000 & 0000 \\ 1010 & 1001 & 1100 & 0000 & 1100 & 0000 \\ 1001 & 1100 & 1010 & 0000 & 1010 & 0000 \\ 0111 & 1000 & 1000 & 1000 & 1000 & 1000 \\ 0000 & 0000 & 1100 & 1100 & 1100 & 1100 \\ 0000 & 0000 & 1010 & 1010 & 1010 & 1010\end{array}\right]$

Equations (7) and (31) together generate $\mathscr{G}_{24}$ [28, fig. 6].

We begin by preparing a table of all 128 coset representatives, written in $+1,-1$ notation, labeling them $\rho^{(0)}, \cdots, \rho^{(127)}$ in some arbitrary order. The beginning of this table (using one obvious ordering) is shown in Table I.

For a typical coset representative

$$
\rho^{(j)}=\left(\rho_{1}^{j}, \cdots, \rho_{24}^{j}\right)
$$

the algorithm will involve the componentwise product

$$
\rho^{(i)} * x=\left(\rho_{1}^{j} x_{1}, \cdots, \rho_{24}^{j} x_{24}\right) \text {. }
$$

TABLE I

128 COSET REPRESENTATIVES FOR $\mathscr{B}$ IN $\mathscr{G}_{24}$, WRITTEN IN $+1,-1$ NOTATION AND ARRANGED In SOME ARbitrary ORder ${ }^{a}$

\begin{tabular}{c|c|c|c|c|c|c}
\hline & I & II & III & IV & V & VI \\
\hline$\rho^{(0)}$ & 1111 & 1111 & 1111 & 1111 & 1111 & 1111 \\
$\rho^{(1)}$ & $\overline{1111}$ & $\overline{11} 11$ & $\overline{1111}$ & $\overline{11} 11$ & 1111 & 1111 \\
$\rho^{(2)}$ & $\overline{1} 1 \overline{11}$ & $\overline{1} 1 \overline{11}$ & $\overline{1} 1 \overline{1} 1$ & $\overline{1} 1 \overline{11}$ & 1111 & 1111 \\
$\rho^{(3)}$ & $1 \overline{111}$ & $1 \overline{111}$ & $1 \overline{111}$ & $1 \overline{111}$ & 1111 & 1111 \\
$\rho^{(4)}$ & 1111 & $\overline{1} 11 \overline{1}$ & $\overline{1111}$ & 1111 & $\overline{11} 11$ & 1111 \\
& $\ldots$ & $\ldots$ & $\ldots$ & $\ldots$ & $\ldots$ & $\ldots$ \\
\hline
\end{tabular}

\footnotetext{
${ }^{a}$ The coordinates are divided into six blocks of four. A bar indicates a negative number.
} 
Inspection of (31) reveals that, in the first block of four coordinates of $\rho^{(\mathrm{i})} * x$, all possible sign combinations of $x_{1}, x_{2}, x_{3}, x_{4}$ occur. The precomputation stage of the algorithm will calculate these 16 combinations in the Gray code order shown in Table III. Similarly in the other five blocks of coordinates, except that in blocks III-VI, the final $x_{i}$ 's (i.e., $x_{12}, x_{16}, x_{20}, x_{24}$ ) only occur with + signs.

We now prepare a second table, the cross-reference table, as follows. For each entry $\rho^{(J)}$ in Table I, we write down for each block of four coordinates where that sign combination is to be found in Table III. For $\rho^{(4)}$, for example, the sign combinations on the six blocks are

$$
\begin{array}{lll}
+-+ & -++- & -+++ \\
+++ & --++ & ++++
\end{array}
$$

which are entries

$\begin{array}{lcc}6 & 14 & 2 \\ 0 & 2 & 0\end{array}$

respectively, of Table III. This gives the fifth line

$$
\chi_{\mathrm{I}}^{4}=6, \chi_{\mathrm{II}}^{4}=14, \chi_{\mathrm{III}}^{4}=2, \cdots, \chi_{\mathrm{VI}}^{4}=0
$$

of Table II.

TABLE II

The Cross-Reference TABLE $\chi_{N}^{j}$ a

\begin{tabular}{c|cccccc}
\hline$j$ & $\mathrm{I}$ & $\mathrm{II}$ & $\mathrm{III}$ & $\mathrm{IV}$ & $\mathrm{V}$ & $\mathrm{VI}$ \\
\hline 0 & 0 & 0 & 0 & 0 & 0 & 0 \\
1 & 2 & 2 & 2 & 2 & 0 & 0 \\
2 & 6 & 6 & 6 & 6 & 0 & 0 \\
3 & 4 & 4 & 4 & 4 & 0 & 0 \\
4 & 6 & 14 & 2 & 0 & 2 & 0 \\
& & & $\ldots$ & & & \\
\hline
\end{tabular}

"An entry $\chi_{N}^{j}=m$ indicates that the sign combination appearing in block $N$ of $\rho^{(i)}$ (in Table I) is to be found in row $m$ of Table III.

\section{The decoding algorithm}

To decode $x=\left(x_{1}, \cdots, x_{24}\right)$.

Precomputation Stage: We compute six Gray code tables, the first of which is shown in Table III. Each entry differs from the previous one in only one coordinate (since the entries are ordered by a Gray code [38], [55], [59]), and so can be computed with just one subtraction. Note also that the entries in the second half of Table III are simply the negatives of the entries in the first half.

Table III is the Gray code table for block I, and involves $x_{1}, \cdots, x_{4}$. The other five tables correspond to blocks II, $\cdots$, VI, and involve $x_{5}, \cdots, x_{8} ; \cdots ; x_{21}, \cdots, x_{24}$, respectively, but have the same sign combinations. However, the last eight rows of the tables for blocks III-VI can be omitted, since $x_{12}, x_{16}, x_{20}$, and $x_{24}$ only occur with + signs. We let $G_{N}(i)$ denote the $i$ th entry in the table for block $N(N=\mathrm{I}, \cdots, \mathrm{VI})$.
TABLE III

Gray Code TABle for Block I, The $i$ Th ENTRy is $G_{\mathrm{I}}(i)$

\begin{tabular}{c|c}
\hline \hline 0 & $x_{1}+x_{2}+x_{3}+x_{4}$ \\
1 & $-x_{1}+x_{2}+x_{3}+x_{4}$ \\
2 & $-x_{1}-x_{2}+x_{3}+x_{4}$ \\
3 & $x_{1}-x_{2}+x_{3}+x_{4}$ \\
4 & $x_{1}-x_{2}-x_{3}+x_{4}$ \\
5 & $-x_{1}-x_{2}-x_{3}+x_{4}$ \\
6 & $-x_{1}+x_{2}-x_{3}+x_{4}$ \\
7 & $x_{1}+x_{2}-x_{3}+x_{4}$ \\
8 & $x_{1}+x_{2}-x_{3}-x_{4}$ \\
9 & $-x_{1}+x_{2}-x_{3}-x_{4}$ \\
10 & $-x_{1}-x_{2}-x_{3}-x_{4}$ \\
11 & $x_{1}-x_{2}-x_{3}-x_{4}$ \\
12 & $x_{1}-x_{2}+x_{3}-x_{4}$ \\
13 & $-x_{1}-x_{2}+x_{3}-x_{4}$ \\
14 & $-x_{1}+x_{2}+x_{3}-x_{4}$ \\
15 & $x_{1}+x_{2}+x_{3}-x_{4}$ \\
\hline &
\end{tabular}

Main Stage: Set record $=0$ and $j^{*}=0$. For $j-0$ through 127 ,

- obtain $\chi_{1}^{j}, \cdots, \chi_{\mathrm{VI}}^{j}$ from Table II;

- obtain $G_{\mathrm{I}}\left(\chi_{\mathrm{I}}^{j}\right), \cdots, G_{\mathrm{VI}}\left(\chi_{V_{\mathrm{I}}}^{j}\right)$ from the Gray code tables, and compute the inner product

$$
\text { ip }=\left|G_{\mathrm{I}}\left(\chi_{\mathrm{I}}^{j}\right)\right|+\cdots+\left|G_{\mathrm{VI}}\left(\chi_{\mathrm{VI}}^{j}\right)\right|
$$

if an even number of the $\left|G_{N}\left(\chi_{N}^{j}\right)\right|$ are even, or

$$
\text { ip }=\left|G_{\mathrm{I}}\left(\chi_{\mathrm{I}}^{j}\right)\right|+\cdots+\left|G_{\mathrm{VI}}\left(\chi_{\mathrm{VI}}^{j}\right)\right|-2 \min _{N}\left|G_{N}\left(\chi_{N}^{j}\right)\right|
$$

otherwise; and

- if ip $>$ record, set record $=\mathrm{ip}$ and $j^{*}=j$.

After the 127th step, a closest codeword in the Golay code to $x$ is

$$
u:=u_{\mathrm{I}} u_{\mathrm{I}} u_{\mathrm{I}} u_{\mathrm{I}} u_{\mathrm{II}} u_{\mathrm{II}} \cdots u_{\mathrm{VI}} u_{\mathrm{VI}} u_{\mathrm{VI}} u_{\mathrm{VI}}
$$

where

$$
u_{N}=\operatorname{sgn}\left(G_{N}\left(\chi_{N}^{j^{*}}\right)\right), \quad N=\mathrm{I}, \cdots, \mathrm{VI},
$$

with the sign of the smallest $\left|G_{N}\left(\chi_{N}^{j^{*}}\right)\right|$ reversed if an even number of the $u_{N}$ are negative. "Record" gives the inner product $u \cdot x$.

The number of steps is roughly 48 for the precomputation stage, plus $12 \cdot 128=1536$ for the main stage, a total of 1584 . To see that this works, we remark that it is just the algorithm of Section II, paragraph 7), modified so as to precompute the $y^{(j)}$ (using the Gray code tables). We have also made use of the special structure of the subcode $\mathscr{B}$ to streamline the calculation of the inner products ip ${ }^{(j)}$ ((32) follows from (11), and (33) from (10)). 


\section{B. The Second Algorithm}

The overall structure of the second algorithm is the same, but now we use the $[24,3]$ subcode $\mathscr{B}$ shown in (4). The 512 coset representatives for $\mathscr{B}$ in $\mathscr{G}_{24}$ are generated by the following matrix:

$\left[\begin{array}{llllll}1111 & 0000 & 1111 & 0000 & 0000 & 0000 \\ 1100 & 1100 & 1100 & 1100 & 0000 & 0000 \\ 1010 & 1010 & 1010 & 1010 & 0000 & 0000 \\ 1010 & 1001 & 1100 & 0000 & 1100 & 0000 \\ 1001 & 1100 & 1010 & 0000 & 1010 & 0000 \\ 1100 & 1010 & 1001 & 0000 & 1001 & 0000 \\ 0111 & 1000 & 1000 & 1000 & 1000 & 1000 \\ 0000 & 0000 & 1100 & 1100 & 1100 & 1100 \\ 0000 & 0000 & 1010 & 1010 & 1010 & 1010\end{array}\right]$

(4) and (34) together generate $\mathscr{G}_{24}$. The precomputation stage computes three Gray code tables, the first containing all 128 combinations

$$
\pm x_{1} \pm \cdots \pm x_{7} \pm x_{8}
$$

the second all 64 combinations

$$
\pm x_{9} \pm \cdots \pm x_{15}+x_{16}
$$

and the third all 64 combinations

$$
\pm x_{17} \pm \cdots \pm x_{23}+x_{24} \text {, }
$$

with an even number of minus signs in each case.

In the main stage $j$ now runs from 0 to 511, and (32a) and (32b) are replaced by the much simpler formula

$$
\text { ip }=\left|G_{\text {I }}\left(\chi_{\mathrm{I}}^{j}\right)\right|+\left|G_{\text {II }}\left(\chi_{\text {II }}^{j}\right)\right|+\left|G_{\text {III }}\left(\chi_{\text {III }}^{j}\right)\right|,
$$

obtained from (6). The final output is

$$
u_{\mathrm{I}} \cdots u_{\mathrm{I}} u_{\mathrm{II}} \cdots u_{\mathrm{II}} u_{\mathrm{III}} \cdots u_{\mathrm{III}} \text {, }
$$

where $u_{N}=\operatorname{sgn}\left(G_{N}\left(\chi^{j}\right)\right), N=\mathrm{I}, \mathrm{II}$, III (cf. (5)). The total number of steps is roughly 192 for the precomputation stage, plus $3 \cdot 512=1536$ for the main stage, a total of 1728.

The Golay code of length 23 can be decoded by a straightforward modification of either algorithm (or alternatively by using the algorithms as they stand and the method of (Section II, paragraph 8)).

\section{A Fasi Decoding Algorithm for the Leech LATTICE}

The Leech lattice $\Lambda_{24}$ is a 24-dimensional lattice that can be defined in a number of ways [10], [15], [17], [23]-[25], [29], [47]-[49], [53], [64], [65]. For example it may be obtained by applying Construction B (see Section II, paragraph 12)) to the Golay code $\mathscr{G}_{24}$, and then doubling the number of points. In [28] we proposed a decoding algorithm for $\Lambda_{24}$ based on the fact that $\Lambda_{24}$ contains $D_{24}$ as a sublattice of index 8192. In view of 10) and 15) in Section II, this algorithm takes about

$$
4 \cdot 24 \cdot 8192=786432
$$

steps to decode one point. In contrast the algorithm given below takes only about 55968 steps, which is about 14 times as fast. This algorithm is based on the "Turyn construction" of $\Lambda_{24}$.

\section{A. The "Turyn Construction" of the Leech Lattice}

Turyn showed around 1965 that the Golay code may be constructed by gluing together three copies of the $[8,4]$ first-order Reed-Muller code (see [50, ch. 18]). The Leech lattice may be constructed in a similar manner by gluing together three copies of the $E_{8}$ lattice. Although this construction has been known for many years, the following particularly simple version has not appeared in print before. We give two sets of coordinates, the first being more elegant, while the second is easier to decode.

Let $\Lambda_{8}$ denote the particular version of $E_{8}$ obtained by multiplying the vectors in (30) by 4 . Typical vectors in $\Lambda_{8}$ are $\left(0^{8}\right),\left( \pm 4, \pm 4,0^{6}\right)$ and $\left( \pm 2^{8}\right)$ with an even number of minus signs.

Definition 1: The Leech lattice $\Lambda_{24}$ consists of the vectors

$$
\left(e_{1}+a+t, e_{2}+b+t, e_{3}+c+t\right),
$$

where $e_{1}, e_{2}, e_{3}$ are arbitrary vectors of $\Lambda_{8}, a, b$ are arbitrary vectors from the list of 16 given in Table $\operatorname{IV}(a)$,

TABLE IV

(a) THE 16 CHOICES FOR $a, b, c$ IN (37), AND $(t)$ THE 16 CHOICES

\begin{tabular}{c|c}
\multicolumn{2}{c}{ FOR $t^{\mathrm{a}}$} \\
\hline (a) & $(\mathrm{t})$ \\
\hline 00000000 & 00000000 \\
40000000 & 22200200 \\
22220000 & 22020002 \\
$\overline{2} 2220000$ & 20220020 \\
22002200 & 02222000 \\
$\overline{2} 2002200$ & 22002020 \\
22000022 & 20202002 \\
$\overline{2} 2000022$ & 20022200 \\
20202020 & $\overline{3} 1111111$ \\
$\overline{2} 0202020$ & $3 \overline{11} 1 \overline{1} 11$ \\
20200202 & $3 \overline{1} \overline{1} 111 \overline{1}$ \\
$\overline{2} 0200202$ & $31 \overline{11} 11 \overline{1} 1$ \\
20022002 & $3111 \overline{1} \overline{111}$ \\
$\overline{2} 0022002$ & $3 \overline{1} 11 \overline{1} \overline{1} 1$ \\
20020220 & $31 \overline{1} 1 \overline{1} 11 \overline{1}$ \\
$\overline{2} 0020220$ & $31 \overline{1} \overline{11} 11$
\end{tabular}

${ }^{a} \mathrm{~A}$ bar indicates a negative number. When multiplied by two these vectors are in $\Lambda_{8}$.

$c$ is the unique vector in Table $\operatorname{IV}(a)$ satisfying

$$
a+b+c \equiv 0\left(\bmod \Lambda_{8}\right) \text {, }
$$

and $t$ is an arbitrary vector from the list of 16 given in Table IV $(t)$.

To see that (37) does define the Leech lattice, we begin with the standard miracle octad generator (MOG) construction of $\Lambda_{24}$ (see [16] or [25], for example), in which the 24 coordinates are divided into three sets of eight. The intersection of $\Lambda_{24}$ with any one of these eight-dimensional spaces is our $\Lambda_{8}$, and the projection onto the same space is $\frac{1}{2} \Lambda_{8}$. The quotient $\frac{1}{2} \Lambda_{8} / \Lambda_{8}$ is an Abelian group of order 256 , and the vectors $a+t, a \in$ Table $\operatorname{IV}(a), t \in$ Table 
IV $(t)$, are coset representatives for $\Lambda_{8}$ in $\frac{1}{2} \Lambda_{8}$. (The blocks of four coordinates in Tables $\operatorname{IV}(a)$ and $\operatorname{IV}(t)$ represent columns of the MOG. See also [16, fig. 27].) The quotient $\Lambda_{24} /\left(\Lambda_{8} \oplus \Lambda_{8} \oplus \Lambda_{8}\right)$ is an abelian group of order 4096, and the vectors

$$
(a+t, b+t, c+t),
$$

with $a, b, c \in$ Table $\operatorname{IV}(a), t \in$ Table $\operatorname{IV}(t)$, and

$$
a+b+c \equiv 0\left(\bmod \Lambda_{8}\right)
$$

are coset representatives for $\Lambda_{8} \oplus \Lambda_{8} \oplus \Lambda_{8}$ in $\Lambda_{24}$.

Definition 1 is based on the version of $E_{8}$ constructed in (30). However, as we saw in Section II, paragraph 13), the version constructed in (24) is easier to decode. To convert from (30) to (24) we multiply by

$$
\frac{1}{2}\left[\begin{array}{rrrrrrrr}
1 & 1 & 0 & 0 & 0 & 0 & 0 & 0 \\
1 & -1 & 0 & 0 & 0 & 0 & 0 & 0 \\
0 & 0 & 1 & 1 & 0 & 0 & 0 & 0 \\
0 & 0 & 1 & -1 & 0 & 0 & 0 & 0 \\
0 & 0 & 0 & 0 & 1 & 1 & 0 & 0 \\
0 & 0 & 0 & 0 & 1 & -1 & 0 & 0 \\
0 & 0 & 0 & 0 & 0 & 0 & 1 & 1 \\
0 & 0 & 0 & 0 & 0 & 0 & 1 & -1
\end{array}\right]
$$

and add $(1,1, \cdots, 1)$. This leads to the second definition of $\Lambda_{24}$.

Let $\Lambda_{8}^{\prime}$ denote the $E_{8}$ lattice in the form in which the 240 minimal vectors consist of 16 of the shape $\left( \pm 4,0^{7}\right)$, and $2^{4} \cdot 14=224$ obtained from

$$
\begin{aligned}
& \text { (2222 0000) } \\
& \left(\begin{array}{ll}
2200 & 2200
\end{array}\right) \\
& \text { (2200 0022) } \\
& \text { (2020 2020) } \\
& \text { (2020 0202) } \\
& \text { (2002 2002) } \\
& \text { (2002 0220) }
\end{aligned}
$$

by inserting arbitrary signs, and by interchanging 0 's and \pm 2 's. Also let $\Lambda_{8}^{\prime \prime}=\mathbf{1}+\Lambda_{8}^{\prime}$, where $\mathbf{1}=(1,1, \cdots, 1)$. Then $\Lambda_{8}^{\prime \prime}$ is precisely the version of $E_{8}$ defincd in (24). Typical vectors in $\Lambda_{8}^{\prime \prime}$ are

$$
\begin{aligned}
& \text { (1111 1111), }
\end{aligned}
$$

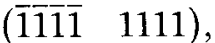

$$
\begin{aligned}
& \text { (1111 } \overline{1} \overline{1} \overline{1}) \text {, }
\end{aligned}
$$

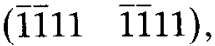

$$
\begin{aligned}
& \text { (i3111 1111), }
\end{aligned}
$$

where the bar indicates a negative number.

Definition 2: The Leech lattice $\Lambda_{24}$ consists of the vectors

$$
1+\left(e_{1}+a+t, e_{2}+b+t, e_{3}+c+t\right)
$$

\begin{tabular}{|c|c|}
\hline (a) & (t) \\
\hline 00000000 & 00000000 \\
\hline 22000000 & $1111 \overline{1111}$ \\
\hline 20200000 & $\overline{1} 1112000$ \\
\hline 20020000 & 20001111 \\
\hline 20002000 & 11021100 \\
\hline 20000200 & 20110011 \\
\hline 20000020 & 11002011 \\
\hline 20000002 & $20 \overline{11} \overline{1100}$ \\
\hline 11111111 & 12101010 \\
\hline$\overline{1111} 1111$ & $21010 \overline{101}$ \\
\hline$\overline{1} 1 \overline{1} 1111$ & $1010210 \overline{1}$ \\
\hline$\overline{1} 11 \overline{1} 1111$ & $210^{-} \overline{1} 010$ \\
\hline$\overline{1} 111 \overline{1} 111$ & 10211001 \\
\hline$\overline{1} 1111111$ & $211001 \overrightarrow{10}$ \\
\hline$\overline{1} 11111 \overline{1} 1$ & $10012 \overline{1} 10$ \\
\hline$\overline{1} 111111 \overline{1}$ & $2 \overline{1} 10 \overline{1001}$ \\
\hline
\end{tabular}

where $e_{1}, e_{2}, e_{3}$ are arbitrary vectors of $\Lambda_{8}^{\prime}, a, b$ are arbitrary vectors from Table $\mathrm{V}(a), c$ is the unique vector in Table V $(a)$ satisfying

$$
a+b+c \equiv 0\left(\bmod \Lambda_{8}^{\prime}\right),
$$

and $t$ is an arbitrary vector from Table $\mathrm{V}(t)$.
TABLE V

(a) THE 16 CHOICES FOR $a, b, c$ IN (43), AND $(t)$ THE 16 CHOICES FOR $t^{\mathrm{a}}$

${ }^{a}$ When multiplied by two these vectors are in $\Lambda_{8}^{\prime}$.

\section{B. Decoding Algorithm for the Leech Lattice $\Lambda_{24}$}

We define $\Lambda_{24}$ by (43). As in Section III-A there are three parts to the algorithm.

The Design Stage: We begin by preparing a list of the 256 sums $a+t, a \in$ Table V $(a), t \in$ Table V $(t)$, labeling them $\rho^{(0)}, \cdots, \rho^{(255)}$ in some arbitrary order. The beginning of this table (using one obvious ordering) is shown in Table VI.

TABLE VI

The 256 Sums $a+t, a \in$ Table $V(a), t \in$ Table $V(t)$, ARRANGED IN SOME ARBITRARY ORDER

\begin{tabular}{c|c}
\hline$\rho^{(0)}$ & 00000000 \\
$\rho^{(1)}$ & 22000000 \\
$\rho^{(2)}$ & 20200000 \\
& $\ldots$ \\
$\rho^{(8)}$ & 11111111 \\
& $\ldots$ \\
$\rho^{(16)}$ & $1111 \overline{1} 111$ \\
$\rho^{(17)}$ & $3311 \overline{1} 111$ \\
& $\ldots$ \\
\hline
\end{tabular}

We now prepare a second table, the cross-reference table, as follows. For each of the 4096 vectors

$$
(a+t, b+t, c+t),
$$

with $a, b, c \in$ Table $\mathrm{V}(a), t \in$ Table $\mathrm{V}(t)$, and satisfying (44), we write down a triple

$$
\chi^{j}=\left(\chi_{1}^{j}, \chi_{2}^{j}, \chi_{3}^{j}\right), \quad 0 \leq j \leq 4095,
$$


TABLE VII

The Cross ReFerence Table

$\overline{\chi^{0}=(0,0,0)}$
$\chi^{1}=(1,1,0)$
$\chi^{2}=(1,0,1)$
$\chi^{3}=(0,1,1)$
$\chi^{4}=(16,16,16)$
$\chi^{5}=(17,17,16)$
$\ldots$

indicating that $a+t$ is entry $\chi_{1}^{j}$ of Table VI, $b+t$ is entry $\chi_{2}^{j}$, and $c+t$ is entry $\chi_{3}^{j}$. In other words

$$
\left(\rho^{\left(x_{i}^{\prime}\right)}, \rho^{\left(x_{2}^{j}\right)}, \rho^{\left(\chi_{3}^{j}\right)}\right)
$$

is a triple (45) that satisfies (44). Part of the cross-reference table based on Table VI is shown in Table VII.

\section{The decoding algorithm}

To decode $x=\left(x_{1}, \cdots, x_{24}\right)$.

Precomputation Stage: In a moment we shall apply the $E_{8}$ decoder of Section II, paragraph 13) to the 256 vectors $\left(x_{1}, \cdots, x_{8}\right)+a+t, a \in$ Table $\mathrm{V}(a), t \in$ Table $\mathrm{V}(t)$.

Before doing this we carry out steps (19)-(21) of the decoder in advance. The components of the vectors $a+t$ range from -2 to +4 . So our first step is to

- compute the $7 \cdot 24=168$ numbers

$$
y_{i m}=x_{i}+m \quad(-2 \leq m \leq 4) \text {, }
$$

- find the nearest integer to $y_{i m}-1$ that is a multiple of four, $4 z_{\text {im }}$ say,

- find the difference $d_{i m}=y_{i m}-4 z_{i m}$,

- if $d_{i m}>2$, change $d_{i m}$ to $2-d_{i m}$, keeping a record of this change, and

- calculate $d_{i m}^{2}$.

The second precomputation step is the most time-consuming part of the algorithm. For $j=0-255$ we calculate

$$
\begin{gathered}
\left(x_{1}, \cdots, x_{8}\right)+\rho^{(j)}, \\
\left(x_{9}, \cdots, x_{16}\right)+\rho^{(j)}, \\
\left(x_{17}, \cdots, x_{24}\right)+\rho^{(j)},
\end{gathered}
$$

and apply the $E_{8}$ decoder of 13) in Section II to these three vectors (making use of the fact that we have already carried out steps (19)-(21) of the algorithm). Let the closest points of $\Lambda_{8}^{\prime \prime}$ to (46)-(48) be

$$
p(j, 1), p(j, 2), p(j, 3),
$$

respectively, and let

$$
d(j, k)=\operatorname{dist}^{2}(x, p(j, k)) .
$$

Main Stage: Set record $=0$ and $j^{*}=0$. For $j=$ $0-4095$, obtain $\chi_{1}^{j}, \chi_{2}^{j}, \chi_{3}^{j}$ from Table VII and calculate the squared distance

$$
d=d\left(\chi_{1}^{j}, 1\right)+d\left(\chi_{2}^{j}, 2\right)+d\left(\chi_{3}^{j}, 3\right) .
$$

If $d<$ record, set record $=d$ and $j^{*}=j$. After the 4095th step, a closest point of $\Lambda_{24}$ to $x$ is

$$
u=\left(p\left(\chi_{1}^{j}, 1\right), p\left(\chi_{2}^{j}, 2\right), p\left(\chi_{3}^{j}, 3\right)\right)
$$

and

$$
\operatorname{dist}^{2}(x, u)=\text { record } .
$$

The number of steps is roughly $4 \cdot 168=672$ for the first precomputation step, $3 \cdot 256 \cdot 56=43008$ for the second, plus $3 \cdot 4096=12288$ for the main stage, a total of 55968 .

To see that this works, we remark that this is the algorithm of Section II, paragraph 15) based on the sublattice $\Lambda_{8}^{\prime} \oplus \Lambda_{8}^{\prime} \oplus \Lambda_{8}^{\prime}$ of index 4096 in $\Lambda_{24}$. The quantity $d$ in (50) is the squared distance from $x$ to a nearest vector in the coset

$$
\mathbf{1}+\Lambda_{8}^{\prime} \oplus \Lambda_{8}^{\prime} \oplus \Lambda_{8}^{\prime}+(a+t, b+t, c+t) .
$$

\section{ACKNOWLEDGMENT}

We thank A. R. Calderbank, A. M. Odlyzko, M. R. Schroeder, and A. D. Wyner for some helpful discussions.

\section{REFERENCES}

[1] Gr. Battail, "Décodage pondéré optimal des codes linéaires en blocs. I. Emploi simplifié du diagramme du trellis," Ann. Télécommun., vol. 38 , pp. $443-459,1983$.

[2] G. Battail, M. C. Decouvelaere, and P. Godlewski, "Replication decoding," IEEE Trans. Inform. Theory, vol. IT-25, pp. 332-345, 1979.

[3] L. D. Baumert and R. J. McEliece, "Performance of some block codes on a Gaussian channel," in Proc. 1975 Int. Telemetering Conf., pp. 189-195.

[4] - " "Soft decision decoding of block codes," Jet Propulsion Lab., Calif. Inst. Tech., Deep Space Network Progress Rep. 42-47, July 1978, pp. 60-64.

[5] L. D. Baumert, R. J. McEliece, and G. Solomon, "Decoding with multipliers," Jet Propulsion Lab., Calif. Inst. Tech., Deep Space Network Progress Rep. 42-34, Aug. 1976, pp: 43-46.

[6] T. Berger, "Optimum quantizers and permutation codes," IEEE Trans. Inform. Theory, vol. IT-18, pp. 759-765, 1972.

[7] T. Berger, F. Jelinek, and J. K. Wolf, "Permutation codes for sources," IEEE Trans. Inform. Theory, vol. IT-18, pp. 160-169, 1972.

[8] E. R. Berlekamp, "The technology of error-correcting codes," Proc. IEEE, vol. 68, pp. 564-593, 1980.

[9] R. E. Blahut, Theory and Practice of Error Control Codes. Reading, MA: Addison-Wesley, 1983, especially p. 482.

[10] I. F. Blake, "The Leech lattice as a code for the Gaussian channel," Inform. Contr., vol. 19, pp. 66-74, 1971.

[11] R. W. D. Booth, M. A. Herro, and G. Solomon, "Convolutional coding techniques for certain quadratic residue codes," in Proc. 1975 Int. Telemetering Conf., pp. 168-177.

[12] P. de Buda, "Encoding and decoding algorithms for an optimal lattice-based code," in IEEE Conf. Rec. 81 CHI648-5, Int. Conf. Commun., June 1981, pp. 65.3.1-65.3.5.

[13] R. de Buda, "The upper error bound of a new near-optimal code," IEEE Trans. Inform. Theory, vol. IT-21, pp. 441-445, 1975.

[14] D. Chase, "A class of algorithms for decoding block codes with channel measurement information," IEEE Trans. Inform. Theory, vol. IT-18, pp. 170-182, 1972.

[15] J. H. Conway, "Three lectures on exceptional groups," in Finite Simple Groups, M. B. Powell and G. Higmann, Eds. New York: 
Academic, 1971, pp. 215-247.

[16] J. H. Conway, "The Golay codes and the Mathieu groups," in The Ieech I.attice, Sphere Packings, and Related Topics, I. H. Conway and N. J. A. Sloane. New York: Springer-Verlag. (in preparation).

[17] J. H. Conway, R. A. Parker, and N. J. A. Sloane, "The covering radius of the Leech lattice," Proc. Royal Soc. London, Ser. A, vol. 380, pp. $261-290,1982$.

[18] J. H. Conway and V. Pless, "On the enumeration of self-dual codes," J. Combinatorial Theory, vol. 28A, pp. 26-53, 1980.

[19] J. H. Conway and N. J. A. Sloane, "On the enumeration of lattices of determinant one," J. Number Theory, vol. 15, pp. 83-94, 1982.

[20] - "The unimodular lattices of dimension up to 23 and the Minkowski-Siegel mass constants," Europ. J. Combinatorics, vol. 3, pp. 219-231, 1982.

[21] , "Voronoi regions of lattices, second moments of polytopes, and quantization," IEEE Trans. Inform. Theory, vol. IT-28, pp. $211-226,1982$

[22] …, "Fast quantizing and decoding algorithms for lattice quantizers and codes," IEEE Trans. Inform. Theory, vol. IT-28, pp. $227-232,1982$.

[23] - "Twenty-three constructions for the Leech lattice," Proc. Roval Soc. London, Ser. A, vol. 381, pp. 275-283, 1982.

[24] - "Lorentzian forms for the Leech lattice," Bull. Amer. Math. Soc., vol. 6, pp. 215-217, 1982.

[25] …, "Laminated lattices," Ann. Math., vol. 116, pp. 593-620, 1982.

[26] - "Complex and integral laminated lattices," Trans. Amer. Math. Soc., vol 280 , pp. 463-490, 1983.

[27] - "A fast encoding method for lattice codes and quantizers," IEEE Trans. Inform. Theory, vol. IT-29, pp. 820-824, 1983.

[28] - " "On the Voronoi regions of certain lattices," SIAM J. Algebraic Discrete Methods, vol. 5, pp. 294-305, 1984.

[29] - - The Leech Lattice, Sphere Packings, and Related Topics. New York: Springer-Verlag, in preparation.

[30] H. S. M. Coxeter, Regular Polytopes, 3rd ed. New York: Dover, 1973.

[31] P. Delsarte, "Partial-optimal piecewise decoding of linear codes," IEEE Trans. Inform. Theory, vol. IT-24, pp. 70-75, 1978.

[32] B. G. Dorsch, "A decoding algorithm for binary block codes and j-ary output channels," IEEE Trans. Inform. Theory, vol. IT-20, pp. 391-394, 1974.

[33] G. S. Evseev, "Complexity of decoding for linear codes" (in Russian), Probl. Peradach. Inform., vol. 19, no. 1, pp. 3-8, 1983. English translation in Probl. Inform. Transmission, vol. 19, no. 1, pp. I-6, 1983.

[34] G. D. Forney, Jr., "Generalized minimum distance decoding," IEEE Trans. Inform. Theory, vol. IT-12, pp. 125-131, 1966.

[35] — " The Viterbi algorithm, "Proc. IEEE, vol. 61, pp. 268-278, 1973.

[36] A. Gersho, "Asymptotically optimal block quantization," IEEE Trans. Inform. Theory, vol. IT-25, pp. 373-380, 1979.

[37] A. Gersho, "On the structure of vector quantizers," IEEE Trans. Inform. Theory, vol. IT-28, pp. 157-166, 1982.

[38] E. N. Gilbert, "Gray codes and paths on the n-cube," Bell Syst. Tech. J., vol. 37, pp. 815-826, 1958.

[39] D. M. Gordon, "Minimal permutation sets for decoding the binary Golay code," IEEE Trans. Inform. Theory, vol. IT-28, pp. 541-543, 1982.

[40] R. R. Green, "A serial orthogonal decoder," JPL Space Programs Summary, vol. 37-39-IV, pp. 247-253, 1966.

[41] —- "Analysis of a serial orthogonal decoder," JPL Space Programs Summary, vol. 37-53-III, pp. 185-187, 1968.

[42] C. M. Hackett, "An efficient algorithm for soft decision decoding of the $(24,12)$ extended Golay code," IEEE Trans. Commun., vol. COM-29, pp. 909-911, 1981 and vol. COM-30, p. 554, 1982.

[43] C. R. P. Hartmann and L. D. Rudolph, "An optimum symbol-bysymbol decoding rule for linear codes," IEEE Trans. Inform. Theory, vol. IT-22, pp. 514-517, 1976.

[44] T. Y. Hwang, "Decoding linear block codes for minimizing word error rate, IEEE Trans. Inform. Theory," vol. IT-25, pp. 733-737, 1979.
[45] - "Efficient optimal decoding of linear block codes," IEEE Trans. Inform. Theory, vol. IT-26, pp. 603-606, 1980.

[46] D. E. Knuth, The Art of Computer Programming, vol. 3. Reading, MA: Addison-Wesley, 1973, p. 216.

[47] J. Leech, "Notes on sphere packings," Canad. J. Math, vol. 19, pp. 251-267, 1967.

[48] J. Leech and N. J. A. Sloane, "Sphere packing and error-correcting codes," Canad. J. Math., vol. 23, pp. 718-745, 1971.

[49] J. I. Lepowsky and A. E. Meurman, "An $E_{8}$-approach to the Leech lattice and the Conway group," J. Algebra, vol. 77, pp. 484-504, 1982.

[50] F. J. MacWilliams and N. J. A. Sloane, The Theory of Error-Correcting Codes. Amsterdam, The Netherlands: North-Holland, 1977.

[51] J. L. Massey, "Foundation and method of channel encoding," in Proc. Int. Conf. on Info. Theory and Systems, NTG-Fachberichte (Berlin), vol. 65, 1978, pp. 148-157.

[52.] H. Miyakawa and T. Kaneko, "Decoding algorithm for error-correcting codes by use of analog weights," Electron. Commun. Japan, vol. 58-A, pp. 18-27, 1975.

[53] H. V. Niemeier, "Definite quadratischer Formen der Dimension 24 und Diskriminante 1," J. Number Theory, vol. 5, pp. 142-178, 1973.

[54] J. Paluszkiewicz, R. Stasinski, and Z. Szymanski, "An algorithm for soft-decision decoding of the Golay and other linear codes," in 1983 IEEE Int. Symp. Information Theory, Abstracts of Papers. New York: IEEE Press, 1983, p. 137.

[55] M. Phister, Jr., Logical Design of Digital Computers. New York: Wiley, 1960, pp. 232-234, 399-401.

[56] V. Pless, "The children of the $(32,16)$ doubly even codes," IEEE Trans. Inform. Theory, vol. IT-24, pp. 738-746, 1978.

[57] V. Pless and N. J. A. Sloane, "On the classification and enumeration of self-dual codes," $J$. Combinatorial Theory, vol. 18, pp. 313-335, 1975.

[58] E. C. Posner, "Combinatorial structures in planetary reconnaissance," in Error Correcting Codes, H. B. Mann, Ed. Ncw York: Wiley, 1969 , pp. $15-46$.

[59] E. M. Reingold, J. Nievergelt, and N. Deo, Combinatorial Algorithms: Theory and Practice. Englewood Cliffs, NJ: PrenticeHall, 1977.

[60] L. D. Rudolph, C. R. P. Hartmann, T. Y. Hwang, and N. Q. Duc, "Algebraic analog decoding of lineary binary codes," IEEE Trans. Inform. Theory, vol. IT-25, pp. 430-440, 1979.

[61] S. S. Ryskov and E. P. Baranovskii, "Solution of the problem of least dense lattice coverings of five-dimensional space by equal spheres" (in Russian), Dokl. Akad. Nauk SSSR, vol,, 222, pp. 39-42, 1975. English translation in Soviet Math. Doklady, vol. 6, pp. $586-590,1975$.

[62] D. Slepian, "Permutation modulation," Proc. IEEE, vol. 53, pp. 228-236, 1965.

[63] N. J. A. Sloane, "Binary codes, lattices and sphere packings," in Combinatorial Surveys, P. J. Cameron, Ed. New York: Academic, 1977, pp. 117-164.

[64] - "A note on the Leech lattice as a code for the Gaussian channel," Inform. Contr., vol 46, pp. 270-272, 1980.

[65] - "Tables of sphere packings and spherical codes," IEEE Trans. Inform. Theory, vol. IT-27, pp. 327-338, 1981.

[66] G. Solomon and H. C. A. van Tilborg, "A connection between block and convolutional codes," SIAM J. Appl. Math., vol. 37, pp. $358-369,1979$.

[67] N. N. Tendolkar and C. R. P. Hartmann, "Generalization of Chase algorithms for soft decision decoding of binary linear codes," IEEE Truns. Inform. Theory, vol. IT-29, pp. 714-721, 1984.

[68] A. J. Viterbi, "Error bounds for convolutional codes and an asymptotically optimum decoding algorithm," IEEE Trans. Inform. Theory, vol. IT-13, pp. 260-269, 1967.

[69] J. K. Wolf, "Efficient maximum likelihood decoding of linear block codes using a trellis," IEEE Trans. Inform. Theory, vol. IT-24, pp. $76-80,1978$

[70] J. Wolfmann, "Nouvelles methodes de decodage du code de Golay $(24,12,8)$," Rev. CETHEDEC Cahier, no. 2, pp. 79-88, 1981.

[71] J. Wolfmann, "A permutation decoding of the $(24,12,8)$ Golay code," IEEE Trans. Inform. Theory, vol. IT-29, pp. 748-750, 1983. 